\title{
Additivity Principle in High-dimensional Deterministic Systems
}

\author{
Keiji Saito ${ }^{1}$ and Abhishek Dhar ${ }^{2}$ \\ ${ }^{1}$ Graduate School of Science, University of Tokyo, 113-0033, Japan \\ ${ }^{2}$ Raman Research Institute, Bangalore 560080, India
}

(Dated: July 3, 2018)

\begin{abstract}
The additivity principle (AP), conjectured by Bodineau and Derrida [Phys. Rev. Lett. 92, 180601 (2004)], is discussed for the case of heat conduction in three-dimensional disordered harmonic lattices to consider the effects of deterministic dynamics, higher dimensionality, and different transport regimes, i.e., ballistic, diffusive, and anomalous transport. The cumulant generating function (CGF) for heat transfer is accurately calculated, and compared with the one given by the AP. In the diffusive regime, we find a clear agreement with the conjecture even if the system is high-dimensional. Surprisingly even in the anomalous regime the CGF is also well fitted by the AP. Lower dimensional systems are also studied and the importance of three-dimensionality for the validity is stressed.
\end{abstract}

PACS numbers: 65.40.Gr,05.40.-a,05.70.Ln,44.10.+i

Understanding the general features of currents and their fluctuations in transport phenomena is one of the main goals in nonequilibrium statistical physics. Heat conduction is a typical transport phenomenon, where one considers heat transferred through a system from a bath at temperature $T_{L}$ to a bath at temperature $T_{R}$. Transport can be classified by the system-size dependent thermal conductivity defined in the linear response regime. Considering a slab of width $W$ and length $N$, for a small $\Delta T=T_{L}-T_{R}$ applied across its length and with $T=\left(T_{L}+T_{R}\right) / 2$ we define

$$
\kappa(T) \equiv \frac{J / W^{2}}{\Delta T / N} \propto N^{\alpha},
$$

where $J$ is the energy current. Fourier's law implies $\alpha=$ 0 , while $\alpha=1$ is ballistic transport. Many dynamical systems with momentum conservation show anomalous transport $(0<\alpha<1)[1-3]$.

In this letter, we consider the properties of current fluctuations beyond the linear response regime in different transport regimes characterized by the parameter $\alpha$. One of the universal properties of current fluctuations which is expected to be valid irrespective of $\alpha$, is the fluctuation theorem [4 7]. It quantitatively connects distribution of positive and negative heat transfer and is valid in the far from equilibrium regime. For diffusive systems, where Fourier's law is satisfied $(\alpha=0)$, some important progress has been made. Bodineau and Derrida [8] made a remarkable conjecture, namely the additivity principle (AP), which enables one to compute all higher orders of current cumulants given just the temperature dependent thermal conductivity $\kappa(T)$ of a system. Bertini and co-workers [9] introduced a macroscopic fluctuation theory (MFT) that describes the asymptotic probability of observing a given time-dependent local current and temperature profile. The MFT is expected to be valid for a wide class of stochastic models, and the AP can be derived from it under the condition that the dominant trajectories are time-independent. From this, the suffi- cient condition to get AP from the viewpoint of the MFT is given by [8]

$$
\kappa(T)\left[\kappa(T) T^{2}\right]^{\prime \prime} \leq[\kappa(T)]^{\prime}\left[\kappa(T) T^{2}\right]^{\prime} .
$$

However, we do not still have the necessary and sufficient condition for the AP. One of the strategies for finding the condition is to test the AP in different concrete models. It was confirmed that the AP is consistent with the exact expressions of several orders of current cumulants in the symmetric simple exclusion process [8]. Recently, the AP was numerically verified in another stochastic system, namely for heat transport in the Kipnis-Marchioro-Presutti model [11], by measuring rare events with a sophisticated algorithm [12]. However, studies so far are concerned only to stochastic processes where not only the reservoir but the system dynamics also is probabilistic. We do not still understand the effects of deterministic system dynamics, higher dimensionality, and non-diffusive transport $(\alpha \neq 0)$. Hence, it is of general interest to consider the AP for systems with bulk Hamiltonian dynamics attached to stochastic thermal reservoirs. In this letter, we for the first time address these effects using the three-dimensional mass disordered harmonic crystal which was recently shown to show different regimes of transport, such as ballistic $(\alpha=1)$, diffusive $(\alpha=0)$, and anomalous transport $(0<\alpha<1)$ [13].

Let $Q$ be the heat transferred from the left reservoir to the system during the measurement time $\tau$, and let $P_{N}\left(q, T_{L}, T_{R}\right)(q \equiv Q / \tau)$ be the distribution of $Q$ for the system with the size $N$. In general, the distribution at large $\tau$ has the large deviation form $P_{N} \sim e^{\tau h_{N}\left(q, T_{L}, T_{R}\right)}$, where $h_{N}$ is the large deviation function (LDF). The AP states that the LDF is given by the sum of the LDFs of subsystems of length $n$ and $N-n: h_{N}\left(q, T_{L}, T_{R}\right)=$ $\max _{T}\left[h_{n}\left(q, T_{L}, T\right)+h_{N-n}\left(q, T, T_{R}\right)\right]$. This conjecture is applied iteratively to break the system into a number of smaller pieces. Using the assumption of local equilibrium for the small pieces one can obtain an explicit expression 
for the LDF of the full system [8]. The cumulant generating function (CGF) defined as

$$
\mu(\lambda)=\lim _{\tau \rightarrow \infty} \frac{1}{\tau} \log \left\langle e^{\lambda Q}\right\rangle
$$

is connected to the LDF through the Legendre transformation $\mu(\lambda)=\max _{q}\left[\lambda q+h_{N}\left(q, T_{L}, T_{R}\right)\right]$ and from this one also gets an expression for the CGF [8]. Verifying the AP prediction through direct simulations of heat conduction in a $3 D$ crystal is extremely difficult. In this letter we use some recent exact results on the CGF of a harmonic crystal to test the AP.

Model and Methods. - We consider a $3 D$ cubic harmonic crystal with a scalar displacement field $x_{n}$ on each lattice site $\boldsymbol{n}=\left(n_{1}, n_{2}, n_{3}\right)$ where $n_{1}=1,2, \ldots, N$ and $n_{2}, n_{3}=1, \ldots W$. The Hamiltonian is given by

$$
\mathcal{H}=\sum_{\boldsymbol{n}} \frac{m_{\boldsymbol{n}} \dot{x}_{\boldsymbol{n}}^{2}}{2}+\frac{k_{0}}{2} x_{\boldsymbol{n}}^{2}+\frac{1}{2} \sum_{\boldsymbol{n}, \hat{\boldsymbol{e}}}\left(x_{\boldsymbol{n}}-x_{\boldsymbol{n}+\hat{\boldsymbol{e}}}\right)^{2},
$$

where $\hat{\boldsymbol{e}}$ denotes unit vectors in the three dimensions. We have set the spring constant between sites to one. Masses are randomly distributed as

$$
m_{n}=1-\Delta \text { or } 1+\Delta
$$

with equal probability. Two faces of the crystal, namely those at $n_{1}=1$ and $n_{1}=N$, have fixed boundary conditions and are coupled to white noise Langevin type heat baths at temperatures $T_{L}$ and $T_{R}\left(<T_{L}\right)$, respectively. In the transverse directions, periodic boundary condition is imposed. Let $\boldsymbol{\ell}$ and $\boldsymbol{r}$ be the sites of left and right faces, namely $\boldsymbol{\ell}=\left(1, n_{2}, n_{3}\right)$, and $\boldsymbol{r}=\left(N, n_{2}^{\prime}, n_{3}^{\prime}\right)$. The equations of motion of the particles are then given by

$$
\begin{aligned}
m_{\boldsymbol{n}} \ddot{x}_{\boldsymbol{n}} & =-k_{0} x_{\boldsymbol{n}}-\sum_{\hat{\boldsymbol{e}}}\left(x_{\boldsymbol{n}}-x_{\boldsymbol{n}+\hat{\boldsymbol{e}}}\right) \\
& -\sum_{\boldsymbol{\ell}} \delta_{\boldsymbol{n}, \boldsymbol{\ell}}\left(\gamma \dot{x}_{\boldsymbol{\ell}}-\eta_{\boldsymbol{\ell}}\right)-\sum_{\boldsymbol{r}} \delta_{\boldsymbol{n}, \boldsymbol{r}}\left(\gamma \dot{x}_{\boldsymbol{r}}-\eta_{\boldsymbol{r}}\right) .
\end{aligned}
$$

The noise terms at different sites are uncorrelated, while at a given site the noise strength is specified by the correlations $\left\langle\eta_{\boldsymbol{\ell}}(t) \eta_{\boldsymbol{\ell}}\left(t^{\prime}\right)\right\rangle=2 \gamma T_{L} \delta\left(t-t^{\prime}\right)$ and $\left\langle\eta_{\boldsymbol{r}}(t) \eta_{\boldsymbol{r}}\left(t^{\prime}\right)\right\rangle=$ $2 \gamma T_{R} \delta\left(t-t^{\prime}\right)$ where we have set the Boltzmann constant to the value one.

We assume that the initial state at $t=0$ is chosen from the steady state distribution . The heat $Q$ flowing from the left reservoir into the system between the times $t=0$ to $t=\tau$ is given by $Q=\sum_{\ell} \int_{0}^{\tau} d t \dot{x}_{\ell}\left(-\gamma \dot{x}_{\ell}+\eta_{\ell}\right)$. The average current $J=\langle Q\rangle / \tau$ in the harmonic crystal is given by a landauer-like formula [10] and this gives the following expression for the size-dependent thermal conductivity defined in Eq. (11):

$$
\begin{aligned}
\kappa & \equiv \frac{J / W^{2}}{\Delta T / N}=\frac{N}{2 \pi W^{2}} \int_{0}^{\infty} d \omega \operatorname{Tr}[\mathcal{T}(\omega)], \\
{[\mathcal{T}(\omega)]_{\ell, \ell^{\prime}} } & =4\left[\boldsymbol{G}^{+} \boldsymbol{\Gamma}_{R} \boldsymbol{G}^{-} \boldsymbol{\Gamma}_{L}\right]_{\boldsymbol{\ell}, \ell^{\prime}} \\
\boldsymbol{G}^{ \pm}(\omega) & =\left[-\boldsymbol{M} \omega^{2}+\boldsymbol{K} \mp \boldsymbol{\Sigma}_{L}(\omega) \mp \boldsymbol{\Sigma}_{R}(\omega)\right]^{-1},
\end{aligned}
$$

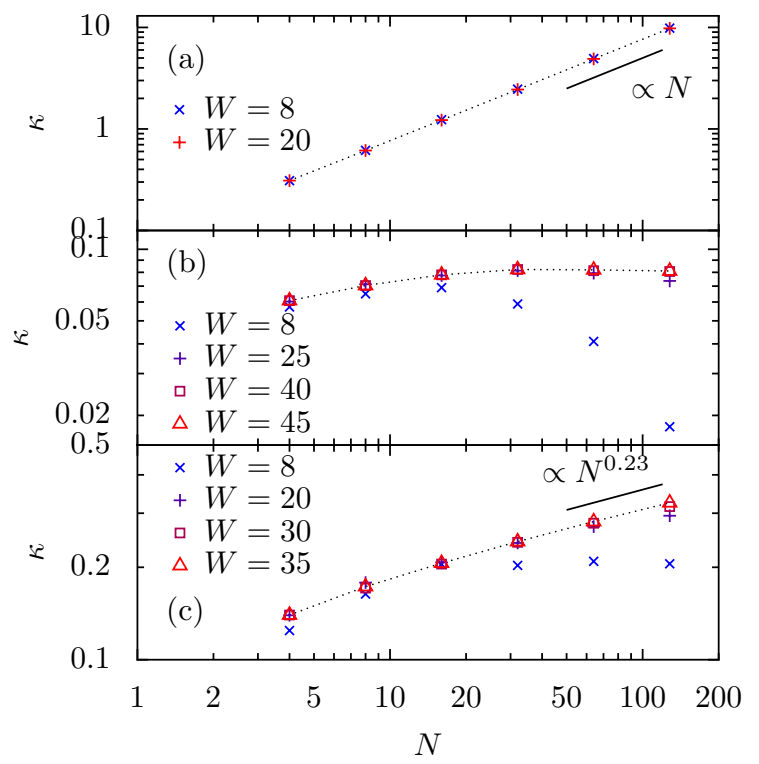

FIG. 1: The size-dependent thermal conductivity $\kappa$ for $\left(\Delta, k_{0}\right)=(\mathrm{a}):(0,0),(\mathrm{b}):(0.22,9.0)$, and $(\mathrm{c}):(0.82,0)$. For sufficiently large $W$ we see respectively (a): ballistic $(\alpha=1),(\mathrm{b})$ : diffusive $(\alpha=0)$, and (c): anomalous transport $(\alpha \sim 0.23)$. Each data-point is obtained from simulations of Eq. (6), while the black dotted line is given by Eq. (7) for largest $W$.

where $\mathcal{T}(\omega)$ is the transmission matrix which describes transmission of phonons emitted from a site on one face attached to a reservoir to a site on another face, and is a $W^{2} \times W^{2}$ matrix. The Green's function $\boldsymbol{G}^{ \pm}(\omega)$ is a $N W^{2} \times N W^{2}$ matrix given by the mass and force constant matrices $\boldsymbol{M}$ and $\boldsymbol{K}$, and the self-energy matrices $\boldsymbol{\Sigma}_{L / R}(\omega)$ whose matrix elements are $\left[\boldsymbol{\Sigma}_{L / R}\right]_{\boldsymbol{n}, \boldsymbol{n}^{\prime}}(\omega)=$ $i \gamma \omega \delta_{\boldsymbol{n}, \boldsymbol{n}^{\prime}} \delta_{\boldsymbol{n}, \boldsymbol{\ell} / \boldsymbol{r}}$. The matrix $\boldsymbol{\Gamma}_{L / R}=\operatorname{Im}\left\{\boldsymbol{\Sigma}_{L / R}(\omega)\right\}$.

Recently it was shown [14] that, not just the current, but an exact expression for the full CGF of the harmonic crystal can be obtained in terms of $\mathcal{T}(\omega)$ and is given by:

$\mu_{H C}(\lambda)=\int_{0}^{\infty} \frac{d \omega}{2 \pi} \operatorname{Tr} \log \left[\mathbf{1}-\mathcal{T}(\omega) T_{L} T_{R} \lambda(\lambda+\Delta \beta)\right]^{-1}$

where $\beta_{L}=T_{L}^{-1}, \beta_{R}=T_{R}^{-1}$ and $\Delta \beta=\beta_{R}-\beta_{L}$. One can verify that the current in Eq. (7) is given by $J=$ $\left.\frac{\partial \mu_{H C}}{\partial \lambda}\right|_{\lambda=0}$. Also Eq. (8) satisfies the fluctuation theorem symmetry relation: $\mu(\lambda)=\mu(-\lambda-\Delta \beta)[5,15]$.

We next discuss the prediction for CGF from the AP, which we will denote by $\mu_{A P}(\lambda)$. In general the CGF can be expressed completely as a temperature integral over the range $\left[T_{R}, T_{L}\right]$ involving the single parameter $\kappa(T)$. For the harmonic case, $\kappa$ is independent of temperature, and explicit expressions can be obtained for $\mu_{A P}(\lambda)$ [1] in terms of a single parameter $\kappa$. These expressions are somewhat lengthy to state and hence we give them in the supplementary material [16]. We note that $\mu_{A P}(\lambda)$ also satisfies the symmetry: $\mu(\lambda)=\mu(-\lambda-\Delta \beta)$.

The main aim of this letter is to compare the AP prediction for $\mu_{A P}(\lambda)$ with the numerical result for $\mu_{H C}(\lambda)$ 


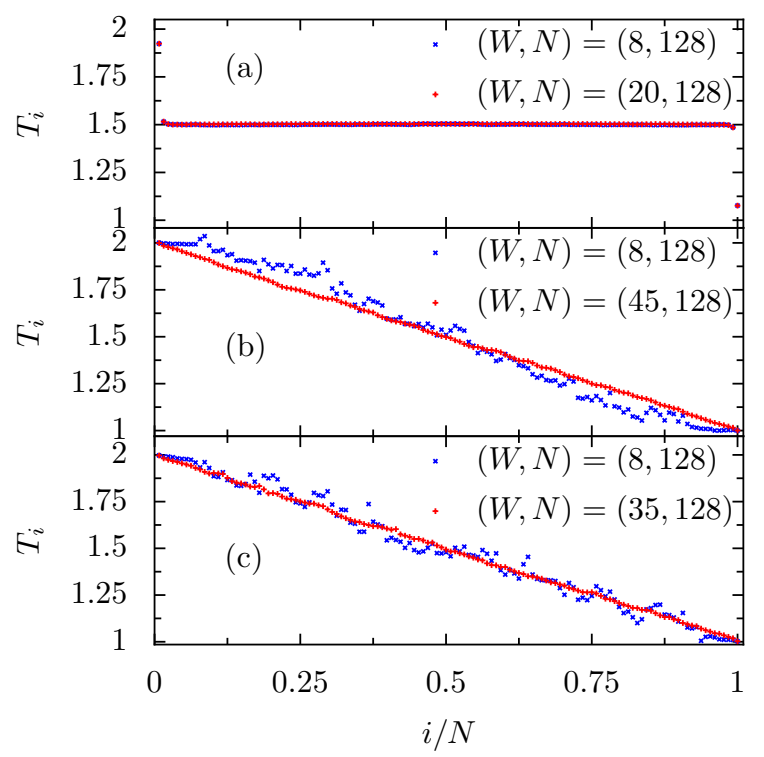

FIG. 2: Temperature profiles for each case in Fig. 1] Temperature is defined as $T_{i}=\sum_{\boldsymbol{n}_{n_{1}}=i}\left\langle m_{\boldsymbol{n}} \dot{x}_{\boldsymbol{n}}^{2}\right\rangle / W^{2}$.

from Eq. (8). We note that for a $3 D$ disordered crystal the heat current depends on the particular realization of disorder, however for large $N$ and $W$ there is self-averaging and sample-to-sample fluctuations become very small. Hence for a fixed disorder strength we get a unique current and $\kappa$ from Eq. (7). This value of $\kappa$ is then used to get $\mu_{A P}(\lambda)$ and compared with $\mu_{H C}(\lambda)$.

Average heat conduction for different regimes. - In $3 D$ disordered systems without pinning potentials $\left(k_{0}=0\right)$, low-frequency extended modes with diverging phonon mean-free-paths exist and lead to anomalous transport. However, a pinning potential removes these modes and transport is then governed by the high-frequency extended diffusive modes. Hence a $3 D$ disordered pinned crystal shows diffusive heat conduction. Based on the results of [13] we expect different regimes of transport and accordingly we chose the following three parameter sets for these regimes: (a) ordered unpinned lattice $\left(\Delta=0, k_{0}=0\right)$ for ballistic transport $(\alpha=1)$, (b) disordered pinned lattice $\left(\Delta=0.22, k_{0}=9.0\right)$ for diffusive transport $(\alpha=0)$, and (c) disordered unpinned lattice $\left(\Delta=0.82, k_{0}=0\right)$ for anomalous transport $(0<\alpha<1)$. To demonstrate the different transport regimes we show the size-dependence of thermal conductivity in Fig. 1 and the typical temperature profiles in Fig. 2. The average heat current was obtained either by direct nonequilibrium simulations of the Langevin equations Eq. (6), or from Eq.(77) using recursive Green's function techniques [13] to evaluate the transmission matrix $\mathcal{T}(\omega)$. Agreement between the two methods is excellent. Each point in Fig. 1 is for one disorder realization and we fixed parameters $\gamma=1, T_{L}=2.0$, and $T_{R}=1.0$.

Remarks on Figs. 1 and 2 are in order. From Fig. 1(a)

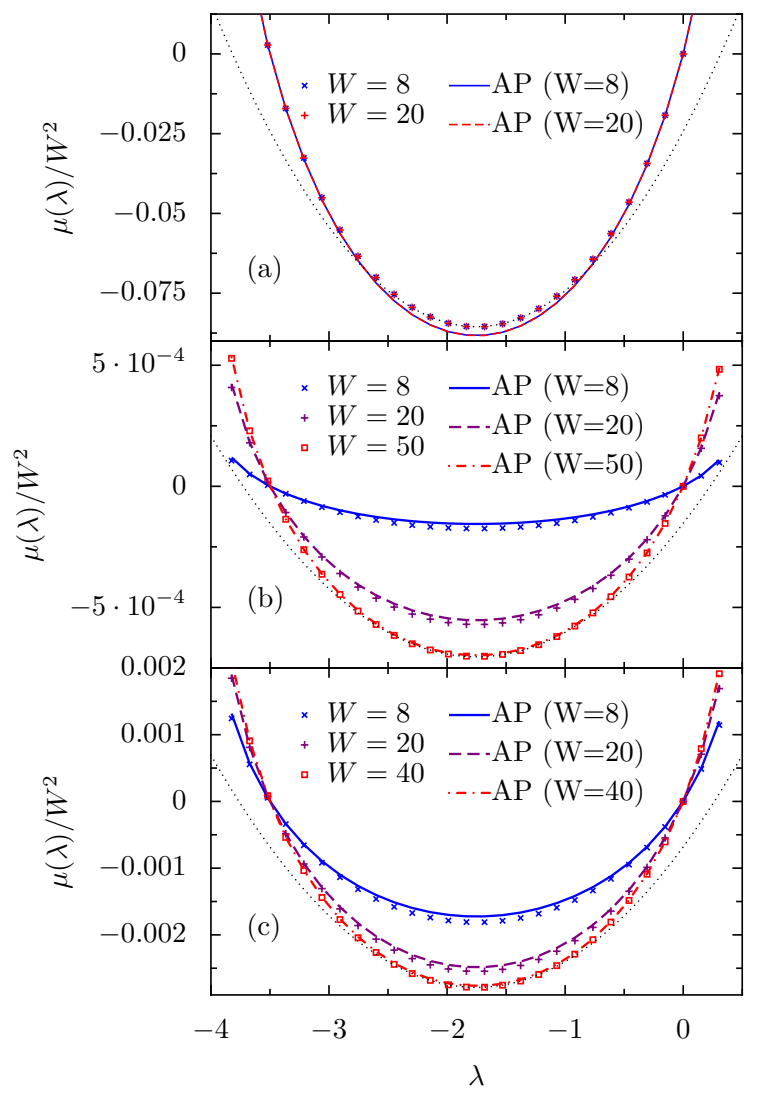

FIG. 3: Comparison of the numerically evaluated CGF $\mu_{H C}(\lambda)$ (points) for a $3 D$ harmonic crystal and the AP predicted curve $\mu_{A P}(\lambda)$ for $N=128$ and different widths. System parameters are the same as in Fig. 1 with $T_{L}=2.0$ and $T_{R}=0.25$. The black dotted line is a quadratic fit to $\mu_{H C}(\lambda)$ for the largest $W$. The range of $\lambda$ is $\left(-\beta_{R}, \beta_{L}\right)$.

we see that $\kappa$ is independent of width and diverges linearly with $N$ implying ballistic transport. On the other hand in Fig. 1(b) and 1(c), we see that for small $W, \kappa$ decreases for increasing $N$. This implies the emergence of phonon localization since the system is quasi-one dimensional. For increasing $W$ with fixed $N$, the data converges to a constant value, which implies the self-averaging effect in disordered systems. Hence, one can get precise $3 D$ behavior for sufficiently large $W$. Fig. 田(b) shows diffusive transport $(\alpha=0)$ for sufficiently large $W$ and $N$, while Fig. 1(c) shows anomalous behavior with systematic power law divergence $(\alpha \approx 0.23)$. The temperature profile in Fig. 2(b) shows clear linear profile consistent with the Fourier's law. Interestingly, even the anomalous case in Fig. 2(c) shows a linear profile which is very different from other nonlinear systems with anomalous transport, such as the Fermi-Pasta-Ulam (FPU) chain which have nonlinear temperature profiles even for small temperature difference between reservoirs [2, 3].

The CGF. - We now present results comparing $\mu_{A P}(\lambda)$ with $\mu_{H C}(\lambda)$ in the three different regimes. We obtained $\mu_{A P}(\lambda)$ by using $\kappa$ from Eq. (7) while $\mu_{H C}(\lambda)$ 
was computed from Eq. (8). In these computations, we set the parameters $\gamma=1.0, T_{L}=2.0, T_{R}=0.25$ and size $N=128$. The results for ballistic, diffusive, and anomalous cases are shown in Figs. 3)(a), 3(b), and 3(c) respectively. Note that both $\mu_{H C}(\lambda)$ in Eq. (8) and $\mu_{A P}(\lambda)$ in [16] exactly satisfy $\mu(0)=\mu(\Delta \beta)=0$. For ballistic case (a), we see deviations from AP curve irrespective of $W$ as expected. On the other hand, in Fig. 3. (b), we see that the agreement between $\mu_{A P}(\lambda)$ and $\mu_{H C}(\lambda)$ improves for increasing $W$. For small $W$ where localization effect is dominant, there are clear deviation from AP curve. For other cases with larger temperature differences, we obtained agreement with the AP for sufficiently large system size [16]. We should also note that the present situation does not satisfy the sufficient condition from the MFT (2), since the thermal conductivity is independent of temperature. Hence, this model extends the sufficiency condition for the AP. Surprisingly, for the case of anomalous transport, we see from Fig. 3. (c) behaviour similar to the diffusive case, with clear verification of AP at sufficiently large $W$. Note that the original theory of the AP conjecture [8] assumes diffusive transport.

The degree of coincidence seen in Fig. 3 is now quantitatively discussed. We note that harmonic lattices, in not only $3 D$, but also in $1 D$ and $2 D$ can show diverging thermal conductivity. For instance, $1 D$ disordered harmonic chains with open boundary condition show diverging thermal conductivity with the power $\alpha=1 / 2$ [17]. Then, one may ask if low dimensional anomalous transport satisfies AP or not. Hence, in addition to $3 D$ cases in Fig. 3] we also discuss low dimensional harmonic systems. We define the following quantity

$$
\delta \equiv\left|\frac{\mu_{H C}\left(\lambda^{*}\right)-\mu_{A P}\left(\lambda^{*}\right)}{\mu_{H C}\left(\lambda^{*}\right)}\right|, \quad \lambda^{*}=-\frac{\Delta \beta}{2},
$$

where $\lambda^{*}$ is the value of $\lambda$ which minimizes $\mu_{A P}(\lambda)$. As seen in Fig. 3, the deviation becomes maximum at the minimum value of the CGF. Hence, the function $\delta$ quantitatively estimates the degree of discrepancy. In Fig. 4 . we show $\delta$ as a function of $W$ for the three cases in $3 D$. Systematic approach to the AP is seen on increasing $W$ for both diffusive and anomalous cases. In the inset, $1 D$ and $2 D$ results for $\delta$ are shown. We consider the system size $N$ for $1 D$ and $N \times N$ for $2 D$ with open boundary condition, and hence, the $x$-axis is $N$ (not $W$ ). For both $1 D$ and $2 D$, results for one realization of random mass are shown. Contrary to what happens in $3 D$, in low dimensions we see no sign of decay of $\delta$, and it remains almost constant value. This implies that the coincidence for anomalous transport is true only for $3 D$ systems.

Discussion. - We have discussed the additivity principle in high-dimensional deterministic systems and considered the effects of deterministic dynamics, dimensionality, and non-diffusive transport $(\alpha \neq 0)$. The AP was originally proposed for $1 D$ diffusive systems. Our main

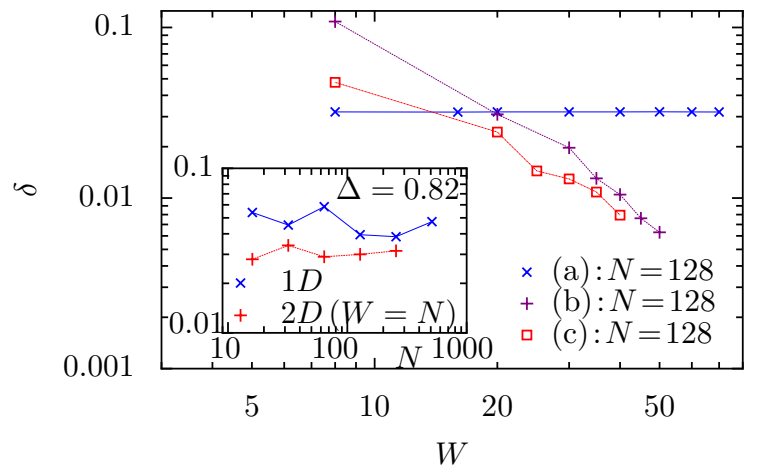

FIG. 4: The quantitative estimation of the degree of agreement of the CGF with AP. The inset shows the results for low-dimensional anomalous cases. $\left(T_{L}, T_{R}\right)=(2.0,0.25)$.

result is to show the validity of $\mathrm{AP}$ in a $3 D$ Hamiltonian system in both the diffusive and anomalous regimes.

In disordered harmonic crystals in $1 D$ and $2 D$ we find that AP is not satisfied for the anomalous cases where $0<\alpha<1$. The major difference between $3 D$ and lower dimensions is that in $3 D$ only a small fraction of the normal modes are localized while in lower dimensions, most of the modes are localized [13, 18]. Hence it is expected that there is no local equilibration in low-dimensions. Then neither the MFT nor the AP are satisfied in these regimes. Our study thus suggests that only the assumptions of (i) local equilibration and (ii) a small current (requiring $\alpha<1$ ) are necessary for the validity of AP. The realization of AP in diffusive and anomalous cases should be related to the time-independence in dominant trajectories in the MFT [9]. A verification for this would be an important future problem, but it is not possible within the present approach, and would require simulations with algorithms as in [12].

The mechanism of anomalous transport in disordered harmonic lattices has some crucial differences from that in low-dimensional nonlinear systems such as FPU chain where Levy type of energy diffusion is seen [19] and temperature profile is always nonlinear. For disordered harmonic crystals the linear temperature profiles suggest that a local response relation $j(x)=-\kappa d T / d x$ is always valid, but with a size-dependent $\kappa$ in the anomalous case. On the other hand levy type of diffusion seen in onedimensional nonlinear models means that the response is non-local (in space) and the AP may need modification. Hence, it will be of great interest to understand the general features of CGF in low-dimensional nonlinear system.

KS was supported by MEXT (23740289). AD thanks DST for support through the Swarnajayanti fellowship. 
[1] F. Bonetto, J.L. Lebowitz, and L. Rey-Bellet, in Mathematical Physics 2000, edited by A. Fokas et. al. (Imperial College Press, London, 2000), p. 128.

[2] S. Lepri, R. Livi, and A. Politi, Phys. Rep. 377, 1 (2003).

[3] A. Dhar, Adv. Phys. 57, 457 (2008).

[4] D. J. Evans, E G. D. Cohen, and G. P. Morriss, Phys. Rev. Lett. 71, 2401 (1993); D. J. Evans and D. J. Searles, Phys. Rev. E 50, 1645 (1994).

[5] G. Gallavotti and E. G. D. Cohen, Phys. Rev. Lett. 74, 2694 (1995)

[6] J. L. Lebowitz and H. Spohn, J. Stat. Phys. 95, 333 (1999).

[7] J. Kurchan, J. Phys. A: Math. Gen. 31, 3719 (1998); G. E. Crooks, Phys. Rev. E 60, 2721 (1999); U. Seifert, Phys. Rev. Lett. 95, 040602 (2005).

[8] T. Bodineau and B. Derrida, Phys. Rev. Lett. 92, 180601 (2004); T. Bodineau and B. Derrida, C. R. Physique 8, 540 (2007).

[9] L. Bertini, A De Sole, D. Gabrieli, G. Jona-Lasinio and C. Landim, Phys. Rev. Lett. 87, 040601 (2001), J. Stat. Phys. 123, 237 (2006).

[10] A. Dhar and D. Roy, J. Stat Phys. 125, 801 (2006); J.-S.
Wang, J. Wang and N. Zeng, Phys. Rev. B 74, 033408 (2006); T. Yamamoto and K. Watanabe, Phys. Rev. Lett. 96, 255503 (2006).

[11] P. I. Hurtado and P. L. Garrido, Phys. Rev. Lett. 102, 250601 (2009), Phys. Rev. E 81, 041102 (2010).

[12] C. Giardiná, J. Kurchan, and L. Peliti, Phys. Rev. Lett. 96, 120603 (2006); C. Giardina, J. Kurchan, V. Lecomte and J. Tailleur, arXiv:1106.4929 (2011).

[13] A. Kundu, A. Chaudhuri, D. Roy, A. Dhar, J. L. Lebowitz, H. Spohn, Europhys. Lett. 90, 40001 (2010); A. Chaudhuri, A Kundu, D. Roy, A. Dhar, J. L. Lebowitz and H. Spohn, Phys. Rev. B 81, 064301 (2010).

[14] K. Saito and A. Dhar, Phys. Rev. E 83, 041121 (2011).

[15] K. Saito and A. Dhar, Phys. Rev. Lett. 99, 180601 (2007).

[16] See supplementary material.

[17] H. Matsuda and K. Ishii, Prog. Theor. Phys. 45, 56 (1970); R. J. Rubin and W. L. Greer, J. Math. Phys. 12, 1686 (1971).

[18] S. R. Nagel, G. S. Grest, and A. Rahman, Phys. Rev. Lett. 53, 368 (1984).

[19] V. Zaburdaev, S. Denisov and P. Hänggi, Phys. Rev. Lett. 106, 180601 (2011).

\section{Supplementary Material for Additivity Principle in High-dimensional Deterministic Systems}

Cumulant generating function from the additivity principle. - We derive the explicit expression of cumulant generating function (CGF) from the additivity principle (AP) for the regime $-\beta_{R} \leq \lambda \leq \beta_{L}$. The AP assumes that the large deviation function (LDF) for the system with the size $N$ is given by [1]

$$
h_{N}\left(q, T_{L}, T_{R}\right)=-\min _{T(x)} \frac{1}{N} \int_{0}^{1} d x \frac{\left[N q+\tilde{\kappa}(T) \frac{d T(x)}{d x}\right]^{2}}{4 T^{2} \tilde{\kappa}(T)},
$$

where $\tilde{\kappa}(T)=W^{2} \kappa(T)$. Variational problem in Eq.(10) is reduced to finding the optimal profile $T(x)$ satisfying [1]

$$
\left(\frac{d T(x)}{d x}\right)^{2}=\frac{(N q)^{2}\left[1+4 K T^{2} \tilde{\kappa}(T)\right]}{\tilde{\kappa}^{2}(T)}
$$

where the function $K$ is determined from the boundary condition $T(0)=T_{L}$ and $T(1)=T_{R}$. From now on, we consider the case of temperature-independent thermal conductivity $\tilde{\kappa}(T)=\tilde{\kappa}$, which is the case for harmonic crystals. We then derive the explicit expression of the CGF. The CGF is given by the Legendre transformation:

$$
\mu(\lambda)=\max _{q}\left[q \lambda+h_{N}\left(q, T_{L}, T_{R}\right)\right] .
$$

Suppose $T_{L}>T_{R}$ and the deviations are not too large so that the optimal profile remains monotonic. Then

$$
\frac{d T(x)}{d x}=\frac{-N q \sqrt{1+4 K T^{2} \tilde{\kappa}}}{\tilde{\kappa}} .
$$

In this case, from the Legendre transformation, the CGF is given by [1]

$$
\begin{aligned}
\mu_{A P}(\lambda) & =-\frac{K}{N}\left[\int_{T_{R}}^{T_{L}} d T \frac{\tilde{\kappa}}{\sqrt{1+4 K T^{2} \tilde{\kappa}}}\right]^{2}, \\
\lambda & =\int_{T_{R}}^{T_{L}} d T \frac{1}{2 T^{2}}\left[\frac{1}{\sqrt{1+4 K T^{2} \tilde{\kappa}}}-1\right] .
\end{aligned}
$$

This expression is valid for $\lambda_{-} \leq \lambda \leq \lambda_{+}$where $\lambda_{ \pm}=$ $\left(\beta_{L}-\beta_{R} \pm \sqrt{\beta_{R}^{2}-\beta_{L}^{2}}\right) / 2$. To go beyond this regime of $\lambda$, we consider a nonmonotonic optimal profile given by

$$
\frac{d T(x)}{d x}= \begin{cases} \pm N q \frac{\sqrt{1+4 K T^{2} \tilde{\kappa}}}{\tilde{\kappa}} & 0 \leq x \leq x_{c} \\ \mp N q \frac{\sqrt{1+4 K T^{2} \tilde{\kappa}}}{\tilde{\kappa}} & x_{c} \leq x \leq 1\end{cases}
$$

where $x_{c}$ satisfies $d T\left(x_{c}\right) / d x=0$ implying $1+$ 
$4 K \tilde{\kappa} T^{2}\left(x_{c}\right)=0$. In these cases, the CGF is given by

$$
\begin{aligned}
\mu_{A P}(\lambda) & =\frac{-K}{N}\left[\int_{T_{L}}^{T\left(x_{c}\right)} d T \frac{\tilde{\kappa}}{\sqrt{1+4 K T^{2} \tilde{\kappa}}}\right. \\
& \left.+\int_{T_{R}}^{T\left(x_{c}\right)} d T \frac{\tilde{\kappa}}{\sqrt{1+4 K T^{2} \tilde{\kappa}}}\right]^{2} \\
\lambda & =\int_{T_{L}}^{T\left(x_{c}\right)} d T \frac{1}{2 T^{2}}\left[1 \pm \frac{1}{\sqrt{1+4 K T^{2} \tilde{\kappa}}}\right] \\
& +\int_{T_{R}}^{T\left(x_{c}\right)} d T \frac{1}{2 T^{2}}\left[-1 \pm \frac{1}{\sqrt{1+4 K T^{2} \tilde{\kappa}}}\right]
\end{aligned}
$$

These expressions are valid for the regime $\lambda_{+} \leq \lambda \leq \beta_{L}$ and $-\beta_{R} \leq \lambda \leq \lambda_{-}$, respectively.

Simplifying Eqs. (14), (15), (17) and (18) is straightforward. By evaluating the integrations we get the following explicit expressions:

$$
\mu_{A P}(\lambda)=\left\{\begin{array}{r}
-\frac{\tilde{\kappa}}{4 N}\left[\log \left(\frac{\sqrt{1+4 \tilde{\kappa} K T_{L}^{2}}+\sqrt{4 \tilde{\kappa} K T_{L}^{2}}}{\sqrt{1+4 \tilde{\kappa} K T_{R}^{2}}+\sqrt{4 \tilde{\kappa} K T_{R}^{2}}}\right)\right]^{2} \\
\cdots \lambda_{-} \leq \lambda \leq \lambda_{+}, \\
\begin{array}{r}
\lambda_{+} \\
\cdots \\
\frac{\tilde{\kappa}}{4 N}\left[\pi-\left(\theta_{L}+\theta_{R}\right)\right]^{2} \leq \lambda \leq \lambda_{-} \\
\lambda_{+} \leq \beta_{L}
\end{array},
\end{array}\right.
$$

where $\theta_{\alpha}(\alpha=L, R)$ is given by

$$
\begin{aligned}
\cos \theta_{\alpha} & =\sqrt{1+4 \tilde{\kappa} K T_{\alpha}^{2}}, \\
\sin \theta_{\alpha} & =\sqrt{4 \tilde{\kappa}|K| T_{\alpha}^{2}} .
\end{aligned}
$$

The function $K$ is given by

$$
\begin{aligned}
K\left(\lambda, T_{L}, T_{R}\right)= & \frac{1}{16 \tilde{\kappa}}\left[\left(\beta_{L}-\beta_{R}-2 \lambda\right)^{2}-2\left(\beta_{L}^{2}+\beta_{R}^{2}\right)\right. \\
& \left.+\left(\frac{\beta_{L}^{2}-\beta_{R}^{2}}{\beta_{L}-\beta_{R}-2 \lambda}\right)^{2}\right] .
\end{aligned}
$$

Effects of small system sizes and large temperature differences. - Since the AP is derived under the assumption of local equilibrium as in Eq.(10), one expects that smaller systems show larger deviation from the AP due to violation of local equilibrium. To see this, we consider $3 D$ systems with cubic $N^{3}$ structure, and calculate the CGF for $N=4,16,32$ with $\left(T_{L}, T_{R}\right)=(2.0,0.25)$. In Fig, the CGF and the deviation $\delta$ defined in the main text are shown for these cases. As expected, $N=4$ clearly shows deviation from the AP curve. As increasing $N$, the deviation $\delta$ decreases.

We next consider the effect of large temperature differences for large systems. For this we obtain the CGF for many temperature sets at fixed large $N$. We note the following exact scaling relation that holds for the CGF :

$$
\mu\left(\lambda, \nu T_{L}, \nu T_{R}\right)=\mu\left(\nu \lambda, T_{L}, T_{R}\right),
$$

where $\nu$ is an arbitrary real number. This holds for both $\mu_{A P}$ and $\mu_{H C}$. From this relation, it is clear that if
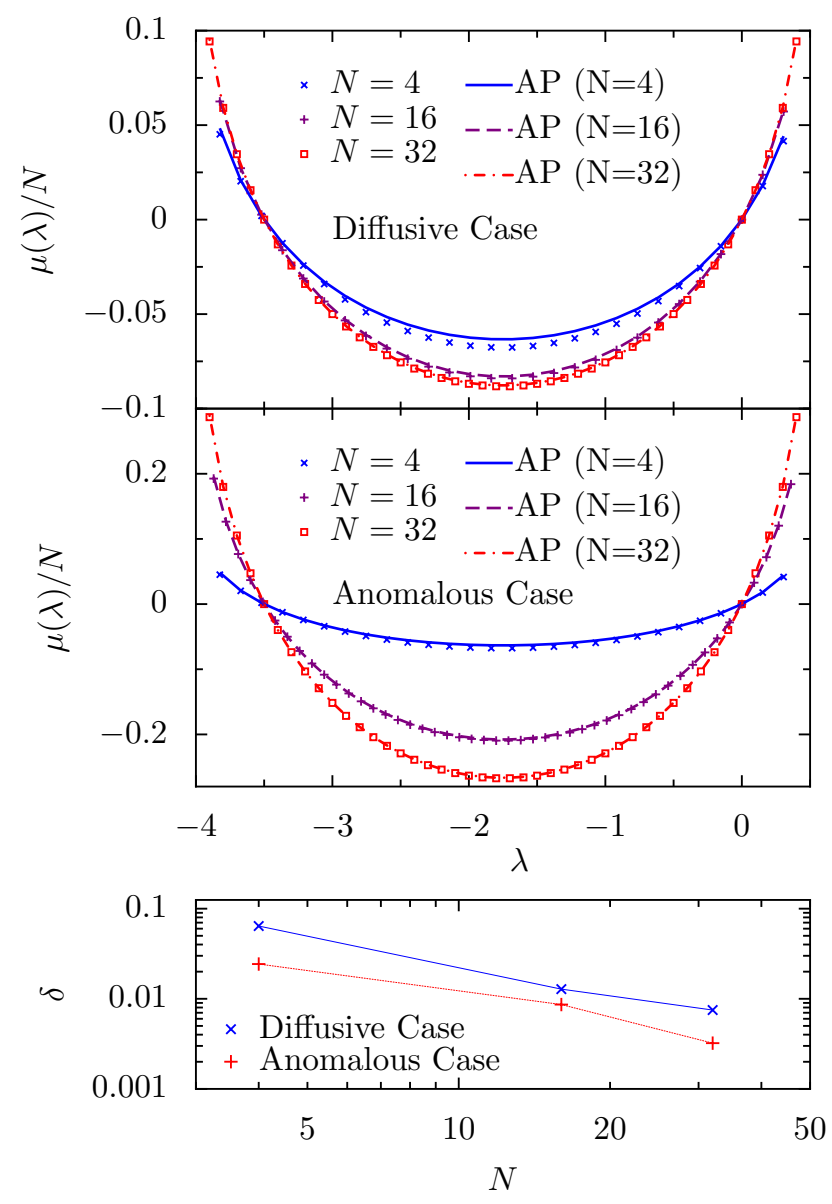

FIG. 5: The CGF in small systems. The system is a cubic structure $N^{3}$.

agreement between the formulas is seen for some temperature difference $\Delta T=T_{L}-T_{R}\left(T_{L}>T_{R}\right)$ and average temperature $T=\left(T_{L}+T_{R}\right) / 2$, then it also holds for $\nu \Delta T$ and $\nu T$. Hence the correct relevant parameter is the ratio $\Delta T / T$. In Fig 6 , we show the CGF of many temperature sets with $N=128$. Points are $\mu_{H C} / W^{2}$, while solid lines are $\mu_{A P} / W^{2}$, and the regime of $\lambda$ is $\lambda \in\left[-\beta_{R}, \beta_{L}\right]$. We see that for the ordered lattice the disagreement with AP becomes large on increasing $\Delta T / T$ while for the disordered case AP is always satisfied. Fig 7 clearly shows that in sufficiently large systems, the AP is accurate over a large range of $\Delta T / T$ and the agreement is better for smaller $\Delta T / T$. The relevance of the parameter $\Delta T / T$ can be roughly understood by considering the criterion for local thermal equilibrium. If the typical mean free path of the heat carriers (phonons here) is denoted by $\ell_{p}$ then the condition for local equilibrium is $\ell_{p}|d T / d x| / T<<1[2]$ or $\Delta T / T<<L / \ell_{p}$. For the ordered ballsitic case $\ell_{p} \sim L$ and so we require $\Delta T / T<<1$. On the other hand for disordered systems $\ell_{p}$ is finite and hence for sufficienlty large size $L$ the condition for local equilibrium is always satisfied for any given $\Delta T / T$. We also note that in diffusive systems the condition for local 


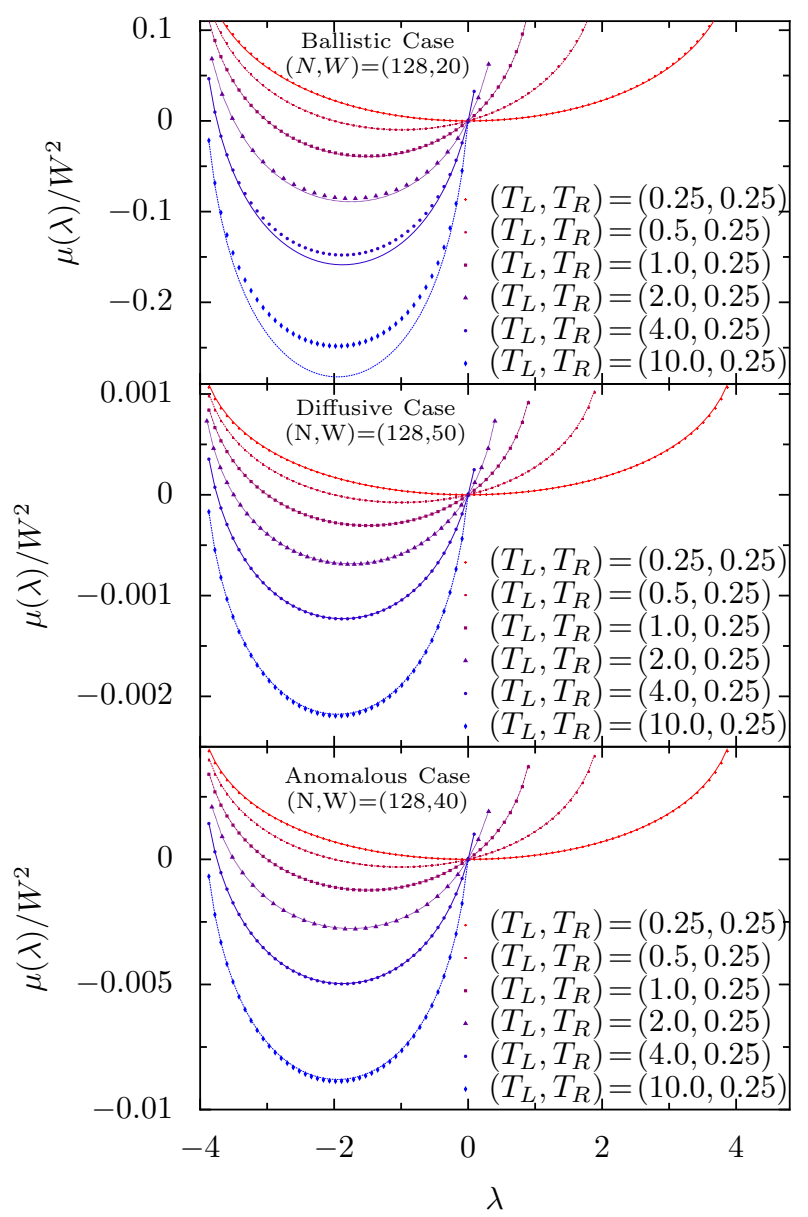

FIG. 6: The CGF in various temperature sets. Points are $\mu_{H C} / W^{2}$, while solid lines are $\mu_{A P} / W^{2}$. The regime of $\lambda$ is $\lambda \in\left[-\beta_{R}, \beta_{L}\right]$

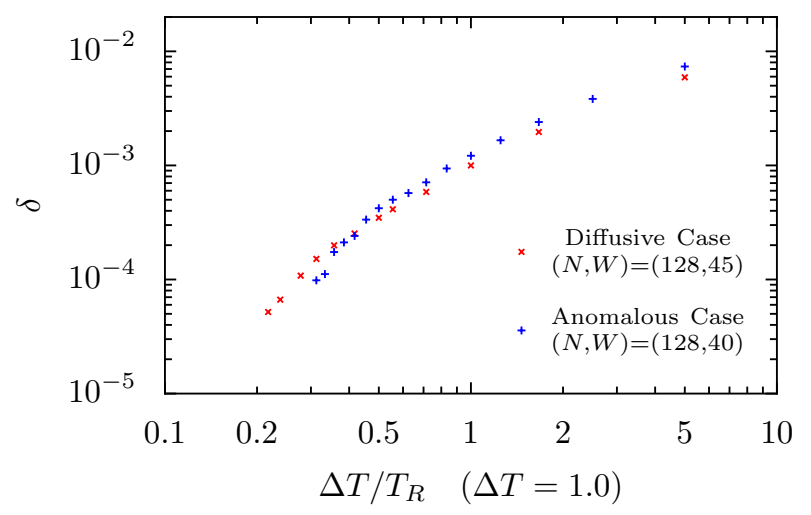

FIG. 7: Deviations from the AP in various temperature regime with fixed temperature difference $\Delta T=T_{L}-T_{R}=1.0$.

equilibrium also ensures that temperature profiles do not show any jumps at the boundaries.

Cumulant generating function for ordered harmonic crystal. - We here give the simplified expression of $\mu_{H C}(\lambda)$ for ordered harmonic crystal $(\Delta=0)$. The sim- plified expression for $\mu_{H C}(\lambda)$ is much more computationally efficient than using the recursive Green's function technique for solving the CGF.

Each lattice is labelled by the vector $\boldsymbol{n}=n_{1} \hat{\boldsymbol{e}}_{1}+n_{2} \hat{\boldsymbol{e}}_{2}+$ $n_{3} \hat{\boldsymbol{e}}_{3}$ where $n_{1}=1, \ldots, N$ and $n_{2}, n_{3}=1, \ldots, W$. Let $U^{(\alpha)}$ be a matrix which acts only on the components in the $\hat{\boldsymbol{e}}_{\alpha}$-direction. We introduce the orthogonal matrix

$$
\begin{aligned}
U & =\mathbf{1}^{(1)} \otimes U^{(2)} \otimes U^{(3)}, \\
U_{m, n}^{(\alpha)} & =\sqrt{\frac{2}{W}} \cos \left(\frac{2 \pi m n}{W}\right), \quad \alpha=2,3 .
\end{aligned}
$$

Using this matrix, scalar variable $x_{\boldsymbol{n}}$ defined on the site $\boldsymbol{n}$ is transformed as

$x_{n_{1}}^{(\boldsymbol{m})}=\frac{2}{W} \sum_{n_{2}, n_{3}} \cos \left(\frac{2 \pi m_{2} n_{2}}{W}\right) \cos \left(\frac{2 \pi m_{3} n_{3}}{W}\right) x_{\boldsymbol{n}}$

where the vector $\boldsymbol{m}$ stands for $\boldsymbol{m}=\left(m_{2}, m_{3}\right),\left(m_{2}, m_{3}=\right.$ $1, \ldots, W)$. By this transformation, the Hamiltonian with $\Delta=0$ is transformed into $W^{2}$ independent chains of the form $\mathcal{H}=\sum_{\boldsymbol{m}} \sum_{n_{1}}\left[\left(\dot{x}_{n_{1}}^{(\boldsymbol{m})}\right)^{2}+x_{n_{1}}^{(\boldsymbol{m})} \boldsymbol{K}_{n_{1}, n_{1}^{\prime}}^{(\boldsymbol{m})} x_{n_{1}^{\prime}}^{(\boldsymbol{m})}\right] / 2$, where $\boldsymbol{K}_{n_{1}, n_{1}^{\prime}}^{(\boldsymbol{m})}$ is the $\boldsymbol{m}$-mode representation of the force matrix $\boldsymbol{K}$ :

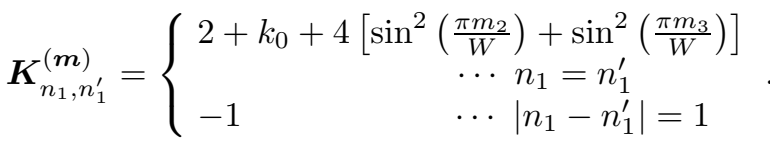

In addition, noise terms preserve the correlations

$$
\begin{aligned}
& \left\langle\eta_{\ell_{1}}^{(\boldsymbol{m})}(t) \eta_{\ell_{1}^{\prime}}^{\left(\boldsymbol{m}^{\prime}\right)}\left(t^{\prime}\right)\right\rangle=2 \gamma T_{L} \delta_{\ell_{1}, \ell_{1}^{\prime}} \delta_{\boldsymbol{m}, \boldsymbol{m}^{\prime}} \delta\left(t-t^{\prime}\right), \\
& \left\langle\eta_{r_{1}}^{(\boldsymbol{m})}(t) \eta_{r_{1}^{\prime}}^{\left(\boldsymbol{m}^{\prime}\right)}\left(t^{\prime}\right)\right\rangle=2 \gamma T_{R} \delta_{r_{1}, r_{1}^{\prime}} \delta_{\boldsymbol{m}, \boldsymbol{m}^{\prime}} \delta\left(t-t^{\prime}\right) .
\end{aligned}
$$

Hence, not only Hamiltonian but also Langevin equations are decomposed into $W^{2}$ independent Langevin dynamics.

We now consider the transmission matrix in terms of which the CGF can be written. The transmission matrix is diagonalized into the $\boldsymbol{m}$-mode

$$
\mathcal{T}^{(\boldsymbol{m})}=4 \gamma^{2} \omega^{2}\left|\boldsymbol{G}_{1, N}^{+(\boldsymbol{m})}\right|^{2} .
$$

with the Green's function given by the inverse of the tridiagonal matrix

$$
\begin{aligned}
{\left[\left[\boldsymbol{G}^{+(\boldsymbol{m})}\right]^{-1}\right]_{n_{1}, n_{1}^{\prime}} } & =-\omega^{2}-\boldsymbol{K}_{n_{1}, n_{1}^{\prime}}^{(\boldsymbol{m})} \\
& -i \gamma \omega \delta_{n_{1}, n_{1}^{\prime}}\left(\delta_{n_{1}, 1}+\delta_{n_{1}, N}\right) .
\end{aligned}
$$

The expression of $\boldsymbol{G}_{1, N}^{+(\boldsymbol{m})}$ is readily obtained, hence, we finally get the explicit formula of $\mu_{H C}(\lambda)$ for ordered harmonic crystal as follows.

$$
\begin{aligned}
\mu_{H C}(\lambda)=- & \frac{1}{2 \pi} \sum_{\boldsymbol{m}} \int_{0}^{\infty} d \omega \log [ \\
& \left.1-\frac{4 \gamma^{2} \omega^{2} \sin ^{2} \theta_{\boldsymbol{m}}}{\left|\Lambda_{\boldsymbol{m}}\right|^{2}} T_{L} T_{R} \lambda(\lambda+\Delta \beta)\right],
\end{aligned}
$$


where

$$
\begin{aligned}
\cos \theta_{\boldsymbol{m}}= & \frac{2+k_{0}-\omega^{2}}{2}+2\left[\sin ^{2}\left(\frac{\pi m_{2}}{W}\right)+\sin ^{2}\left(\frac{\pi m_{3}}{W}\right)\right] \\
\Lambda_{\boldsymbol{m}}= & {\left[\left(1-\gamma^{2} \omega^{2}\right) \cos \theta_{\boldsymbol{m}}-2 i \gamma \omega\right] \sin \left(N \theta_{\boldsymbol{m}}\right) } \\
& +\left(1+\gamma^{2} \omega^{2}\right) \sin \theta_{\boldsymbol{m}} \cos \left(N \theta_{\boldsymbol{m}}\right) .
\end{aligned}
$$

[1] T. Bodineau and B. Derrida, Phys. Rev. Lett. 92, 180601 (2004); T. Bodineau and B. Derrida, C. R. Physique 8
$540(2007)$

[2] H. J. Kreuzer, Nonequilibrium thermodynamics and its statistical foundations (Oxford university press, 1981). 